\title{
Enhanced Region based Clustering using Hybrid Approach of Mobile Sink and Fuzzy Logic Tool for Wireless Sensor Network
}

\author{
Amneet Kaur \\ Computer Science and Engineering \\ LLRIET, Moga, India
}

\author{
Harpreet Kaur \\ Computer Science and Engineering \\ LLRIET, Moga, India
}

\begin{abstract}
Wireless sensor network has revolutionized the way computing and software services are delivered to the clients on demand. It consists of number of sensors called nodes and a base station. Nodes collect data and send to the base station. The nodes are connected with each other without a wired connection. There are number of nodes which send data at a time and because of that numbers of problems occur. For this, nodes are divided into cluster and a cluster head will be formed.WSN is a battery powered system and when the battery dies no data send or received. So when all nodes participate for sending and receiving data then system dies early. When the energy of the cluster head is less, then next cluster head will be formed and at one time, one cluster head is formed. Cluster head collect data from nodes and then send to the base station. WSN is also used in many applications like, constant monitoring \& detection of specific events, military and battlefield surveillance, forest fire and flood detection, habitat exploration of animals, patient and home appliances. This research work proposed a new method for cluster head selection having less computational complexity. It was also found that the modified approach has improved performance to that of the other clustering approaches. In this work, the network area is divided into same sized small regions. Sensor nodes are randomly deployed in each predefined sub-area where each region will have its region head $(\mathrm{RH})$ and multiple member nodes. The member nodes in a specific region will send the data to the RH. RH within the region will be elected by distributed mechanism and will be based on fuzzy variables. The Region heads are divided into 2 different classes boundary RH and non-boundary RH. Only boundary RH will send the data to the BS only when it will come within its region and Non-boundary region heads will evaluate the lower bound limit and upper bound limit of both $\mathrm{x}$-axis and $\mathrm{y}$-axis to evaluate the next relay node. It will choose the relay node on the path where BS is closely located which will reduce the transmission delay and will also reduce the data packet size. It was found that the proposed algorithm gives a much improved network lifetime as compared to existing work. Based on our model, transmission tuning algorithm for cluster-based WSNs has been proposed to balance the load among cluster heads that fall in different regions. This algorithm is applied prior to a cluster algorithm to improve the performance of the clustering algorithm without affecting the performance of individual sensor nodes. The proposed work is validated using throughput, average energy consumption, average remaining energy and network delay metrics.
\end{abstract}

\section{Keywords}

Wireless Sensor Network (WSN), Region Head, Base Station, Fuzzy Logic.

\section{INTRODUCTION}

A Wireless Sensor Network (WSN) consists of various sensor nodes and at least one base station. Sensor nodes are the main component of sensor network. These nodes have the capability to collect data, analyze them and route them to a sink point i.e. a sensor is a device which senses the information and pass it on to base station. Sensors are typically used to measure the changes in physical environmental parameters like temperature, pressure, humidity, sound, vibration and changes in the health parameter of person e.g. blood pressure and heartbeat. The measured parameters are stored at base station and also are available for the end user.

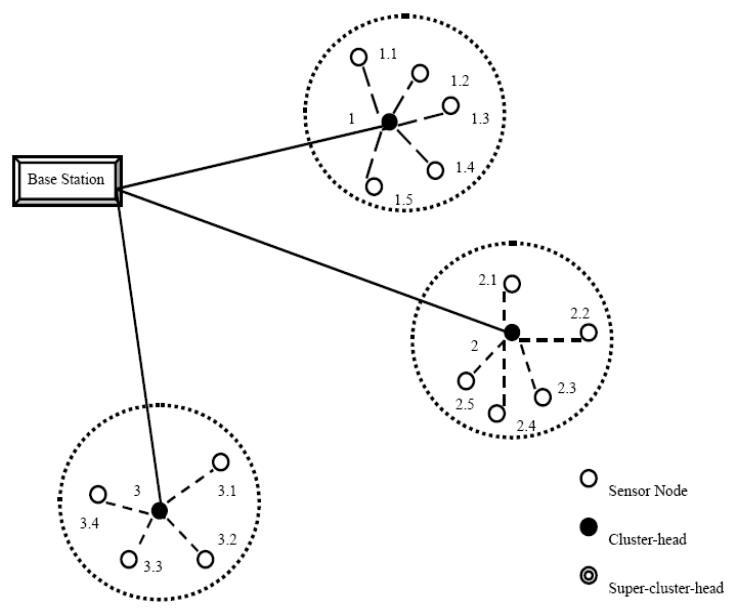

Figure 1: General Architecture of Sensor Network

Figure 1 describes the general architecture of sensor network that consists of various sensor nodes and these sensor nodes sends the data to cluster head and then these cluster heads of each region pass that data to the base station. These sensor nodes are deployed randomly in the network. Clustering is one of the design methods used to manage the network energy consumption efficiently, by minimizing the number of nodes that take part in long-distance communication with the base station and distributing the energy consumption evenly among the nodes in the network. In Clustering, the sensor nodes are apportioned into distinctive groups. Each one cluster is overseen by a node alluded as group head $(\mathrm{CH})$ and different nodes are alluded as cluster nodes. Group nodes don't speak straightforwardly with the sink node. They need to pass the gathered information to the cluster head. Group head will total the information, gained from cluster nodes and transmits it to the base station. In this way it minimizes number of messages imparted to base station. 


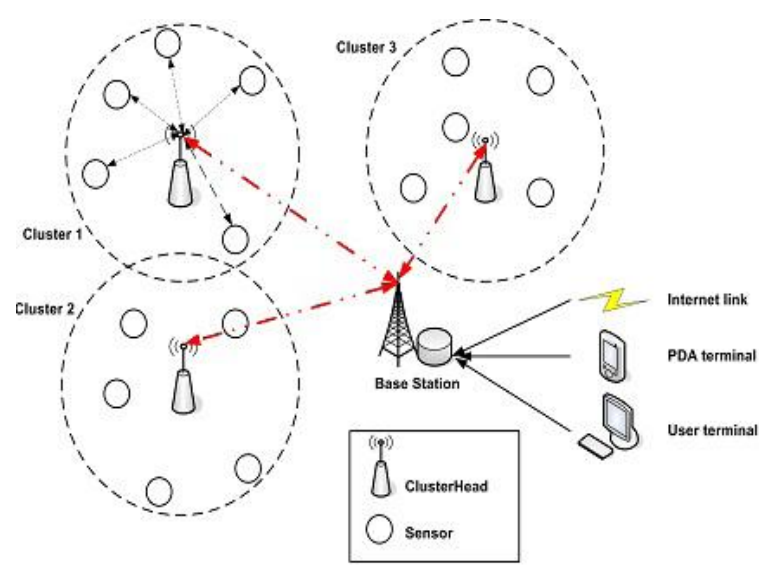

Figure 2: Clustering in WSN

It may be that sometimes WSN is firstly divided into regions and then clustering is performed and this is known as region based clustering. It involves grouping of sensor nodes into clusters \& electing cluster head for all clusters. Also that base station may be kept static or it may move to gather the data. The cluster head can be selected using fuzzy logic. Fuzzy Logic (FL) is a method of reasoning that resembles human reasoning. The approach of FL imitates the way of decision making in humans that involves all intermediate possibilities between digital values YES and NO. Like we aggregate data and form a number of partial truths which we aggregate further into higher truths which in turn, when certain thresholds are exceeded, cause certain further results such as motor reaction. A similar kind of process is used in neural networks, expert systems and other artificial intelligence applications. Various fuzzy variables can used to select the cluster head. Sometimes mobile sink is used. Mobile sink means that base station is movable. Random mobility and controlled mobility models are used in mobile sink. In random mobility the sink is moved randomly within the network and when the sink is deterministically moved across the network it is referred as controlled mobility.

\section{RELATED WORK}

Dastgheib, Oulia and Ghassami (2011) proposed an efficient method for clustering using fuzzy logic with appropriate inputs and combine it with the good features of LEACH. This method was fully distributed. Therefore its speed was more and its energy consumption was less than centralized methods. Also proposed method will resolve the weaknesses of LEACH and more efficient than the existing methods.Sharma and Kumar (2012) proposed F-MCHEL, a homogeneous energy protocol. In LEACH protocol the clusters were formed randomly on the basis of threshold values; whereas, in the proposed protocol a fuzzy logic approach was used to elect the cluster-head based on two descriptors - energy and proximity distance .Out of these elected cluster heads one Master cluster head had been elected .The cluster head which had the maximum residual energy was elected as Master cluster head. In conventional Leach approach all the Cluster heads were used to send the aggregated information to the base station, however in the proposed protocol only Master cluster head sends the aggregated information to the base station. Simulation results on MATLAB shows that the proposed protocol provides higher energy efficiency, better stability period and lower instability period as compared to LEACH protocol in spite of overhead of election of Master cluster head. Results obtained shown that an appropriate Master cluster-head election can drastically reduce the energy consumption and enhance the lifetime of the network. Beiranvand, Patooghy and Fazeli (2013) proposed an energy efficient routing algorithm which saved a significant portion of inner-network communications energy. The proposed routing algorithm selects sensor nodes with higher residual energy, more neighbors, and lower distance from the Base Station (BS) as Cluster Head (CH) nodes. Then, it managed sensor nodes appropriately and constructs clusters such a way to maximize WSN lifetime and minimize average energy dissipation per each sensor node. To evaluate the proposed routing algorithm, various simulations had been carried out by using of MATLAB simulator. The proposed routing algorithm was compared to the previous proposed algorithms e.g., LEACH, DBS, and LEACH-C algorithms. Results of the simulations shown that the proposed routing algorithm improved the WSN performance at least $65 \%$, reduces the energy consumption of the WSN up to $62 \%$, and improves the successfully delivered packet ratio by at least $56 \%$ as compared to the previous routing algorithms. Pavithra, Shivashankar and Poornima (2016) introduced a Rendezvous design technique, where some sensor nodes were selected as an RP's and the nodes which were not RP's will forward the data to the nearest RP's. The major issue was to find the set of RP's and determine the tour that visits these RP's. To overcome this issue, some weight based on the number of packets that it sends and the nearest hop distance.

\section{PROPOSED WORK}

In the proposed work, the whole network is divided into small-small regions. The sensor nodes are randomly deployed in these sub-areas. Each region has its region head. The regions along the boundary have boundary region head and the regions that are not along the boundary have the nonboundary region heads. The member nodes send their data to the region heads.

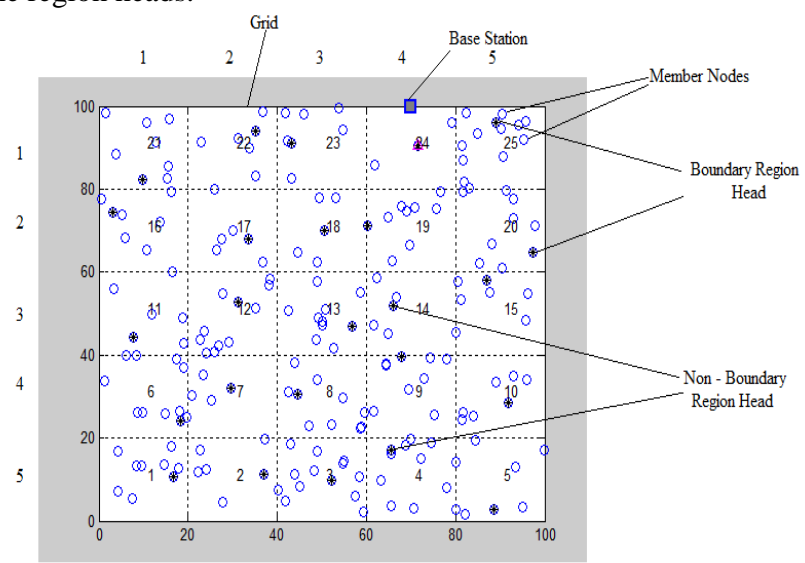

Figure 3: Region Based Clustering

\subsection{Algorithm for the Proposed Work}

The various steps that are followed are as follows:

i. The network area is of $\mathrm{n} \times \mathrm{n}$ meters.

ii. Divide this network area into same sized smallsmall regions (in meters each).

iii. All nodes will send their energy level and location information to the sink

iv. Sensor nodes are randomly deployed in each predefined sub-area. Each region will have its region head $(\mathrm{RH})$ and multiple member nodes. The member nodes in a specific region will send the data to the RH.

v. RH within the region will be elected by 
distributed mechanism and will be based on fuzzy variables like highest residual energy, base station distance and more number of neighbors.

vi. Each RH will create a TDMA schedule for the member nodes to transmit the data.

vii. Base station is movable along the boundary.

viii. $\quad$ BS is not energy constrained. It means it is having sufficient amount of energy for retrieving the data from the region heads and movement along the boundary.

ix. The Region heads are divided into 2 classes:

Boundary RH

Non-Boundary RH

x. Only boundary RH will send the data to the BS only when it will come within its region.

xi. Boundary RH will have sufficient amount of time to send the data to the base station when BS is within its boundary.

xii. When boundary RH is sending its data to the BS, the other nodes will not send the data to that specific boundary RH.

xiii. Non-boundary region heads will evaluate the lower bound limit and upper bound limit of both $\mathrm{x}$-axis and $y$-axis to evaluate the next relay node. It will choose the relay node on the path where BS is closely located which will reduce the transmission delay and will also reduce the data packet size.

Table 1: Fuzzy Rule Base System

\begin{tabular}{|c|c|c|c|c|}
\hline S.No & $\begin{array}{c}\text { Residual } \\
\text { Energy }\end{array}$ & $\begin{array}{c}\text { Base } \\
\text { Station } \\
\text { Distance }\end{array}$ & $\begin{array}{l}\text { Number of } \\
\text { Neighbors }\end{array}$ & $\begin{array}{c}\text { Chance to } \\
\text { select } \\
\text { Region } \\
\text { Head } \\
\end{array}$ \\
\hline 1 & Low & Close & Low & Medium low \\
\hline 2 & Low & Close & Medium & $\begin{array}{l}\text { Very high } \\
\text { medium }\end{array}$ \\
\hline 3 & Low & Close & High & $\begin{array}{c}\text { Very low } \\
\text { high }\end{array}$ \\
\hline 4 & Low & Medium & Low & $\begin{array}{c}\text { Low } \\
\text { medium }\end{array}$ \\
\hline 5 & Low & Medium & Medium & Medium \\
\hline 6 & Low & Medium & High & $\begin{array}{c}\text { Moderately } \\
\text { low high }\end{array}$ \\
\hline 7 & Low & Far & Low & $\begin{array}{c}\text { Very very } \\
\text { low }\end{array}$ \\
\hline 8 & Low & Far & Medium & Very low \\
\hline 9 & Low & Far & High & $\begin{array}{c}\text { Relatively } \\
\text { medium low }\end{array}$ \\
\hline 10 & Medium & Close & Low & $\begin{array}{l}\text { Very low } \\
\text { medium }\end{array}$ \\
\hline 11 & Medium & Close & Medium & $\begin{array}{l}\text { Moderately } \\
\text { low medium }\end{array}$ \\
\hline 12 & Medium & Close & High & $\begin{array}{l}\text { Relatively } \\
\text { low high }\end{array}$ \\
\hline 13 & Medium & Medium & Low & $\begin{array}{c}\text { Relatively } \\
\text { low medium }\end{array}$ \\
\hline 14 & Medium & Medium & Medium & $\begin{array}{c}\text { Rather } \\
\text { medium }\end{array}$ \\
\hline 15 & Medium & Medium & High & $\begin{array}{l}\text { Relatively } \\
\text { high } \\
\text { medium }\end{array}$ \\
\hline 16 & Medium & Far & Low & Rather low \\
\hline 17 & Medium & Far & Medium & High low \\
\hline 18 & Medium & Far & High & $\begin{array}{c}\text { Very high } \\
\text { low }\end{array}$ \\
\hline
\end{tabular}

\begin{tabular}{|c|c|c|c|c|}
\hline 19 & High & Close & Low & Low high \\
\hline 20 & High & Close & Medium & $\begin{array}{c}\text { High } \\
\text { medium }\end{array}$ \\
\hline 21 & High & Close & High & $\begin{array}{c}\text { Very very } \\
\text { high }\end{array}$ \\
\hline 22 & High & Medium & Low & Rather high \\
\hline 23 & High & Medium & Medium & High \\
\hline 24 & High & Medium & High & Very high \\
\hline 25 & High & Far & Low & Low \\
\hline 26 & High & Far & Medium & $\begin{array}{c}\text { Relatively } \\
\text { high low }\end{array}$ \\
\hline 27 & High & Far & High & $\begin{array}{c}\text { Medium } \\
\text { high }\end{array}$ \\
\hline
\end{tabular}

\subsection{Work Flow}

The following flowchart represents the whole working :

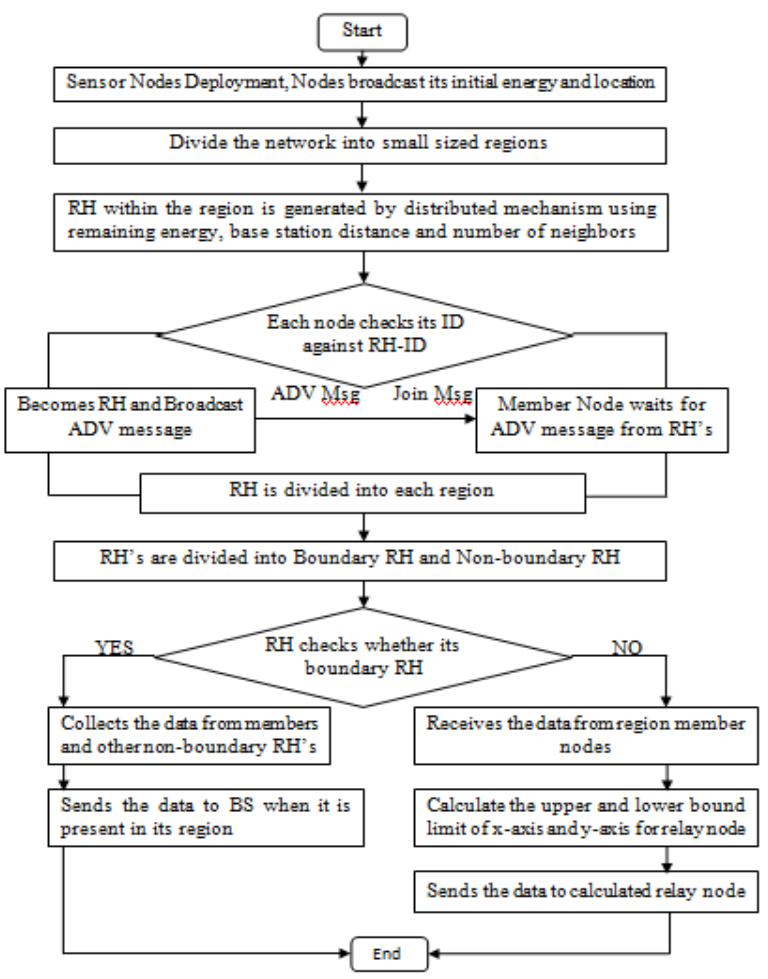

Figure 4: Flow of Work

\section{RESULTS AND DISCUSSION}

The proposed work has been done in MATLAB. The parameters required for simulation are as follows:

Table 2: Simulation Parameters

\begin{tabular}{|c|c|}
\hline Network Parameters & Values \\
\hline Network Size & $100 \mathrm{~m} * 100 \mathrm{~m}$ \\
\hline Number of Sensor Nodes & 200 \\
\hline Initial Energy of Sensor Nodes & $0.5 \mathrm{~J}$ \\
\hline $\begin{array}{c}\text { Packet Size } \\
\text { consumption }\end{array}$ & $4000 \mathrm{bits}$ \\
\hline
\end{tabular}




\begin{tabular}{|c|c|}
\hline $\begin{array}{c}\text { Amplification Energy (Cluster to } \\
\text { BS)d }>=\mathrm{d} 0\end{array}$ & Efs $=10 \mathrm{pJ} / \mathrm{bit} / \mathrm{m} 2$ \\
\hline $\begin{array}{c}\text { Amplification Energy (Cluster to } \\
\text { BS)d }<=\text { do }\end{array}$ & Emp $=0.0013 \mathrm{pJ} / \mathrm{bit} / \mathrm{m} 2$ \\
\hline
\end{tabular}

The network area is of $100 * 100 \mathrm{~m}$ and it is divided into small regions of $20 * 20 \mathrm{~m}$ each. Each region has its own cluster head. The base station is movable and it will move along the boundary of the network. The region heads are divided into two classes namely Boundary Region head and NonBoundary region heads. Boundary RH will send the data to the BS only when it will come within its region. When boundary RH is sending its data to the BS, the other nodes will not send the data to that specific boundary RH and Nonboundary region heads will evaluate the lower bound limit and upper bound limit of both $\mathrm{x}$-axis and $\mathrm{y}$-axis to evaluate the next relay node. It will choose the relay node on the path where BS is closely located. Below is the figure representing the network simulation.

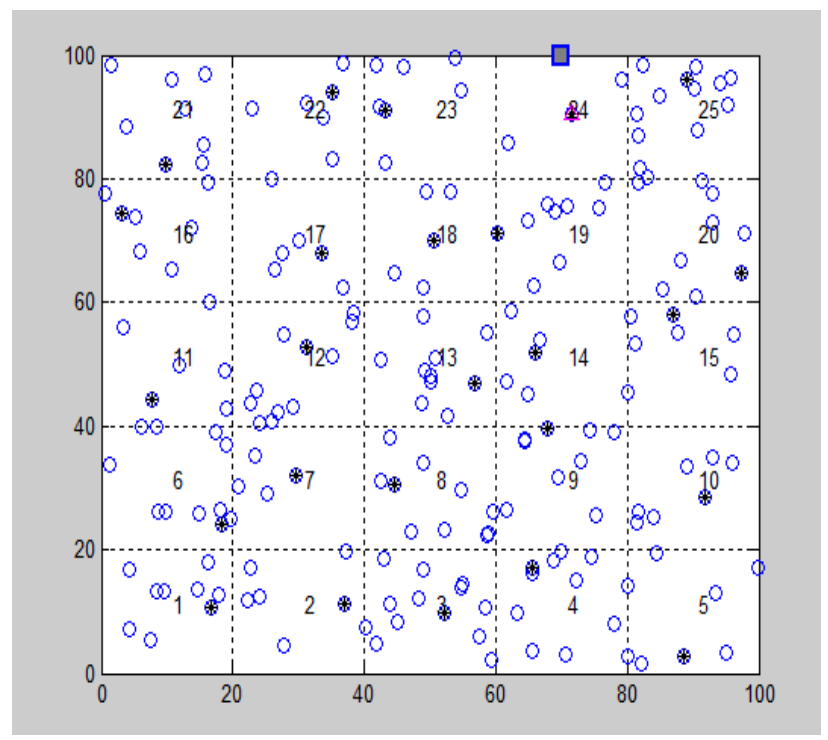

Figure 5: Simulation of Network

The following figure represents the network with all the nodes alive. In the beginning, all the nodes are alive.

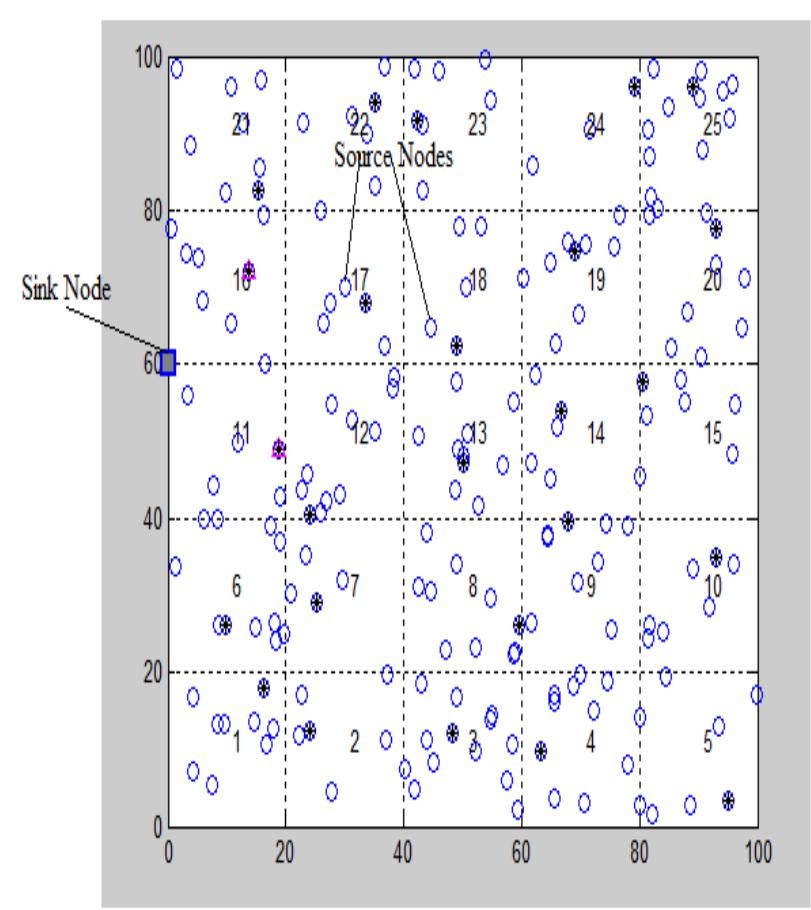

Figure 6: Network when nodes are Alive

With the passage of time energy of nodes gets decreases so they die. The following figure represents the network where some nodes are dead.

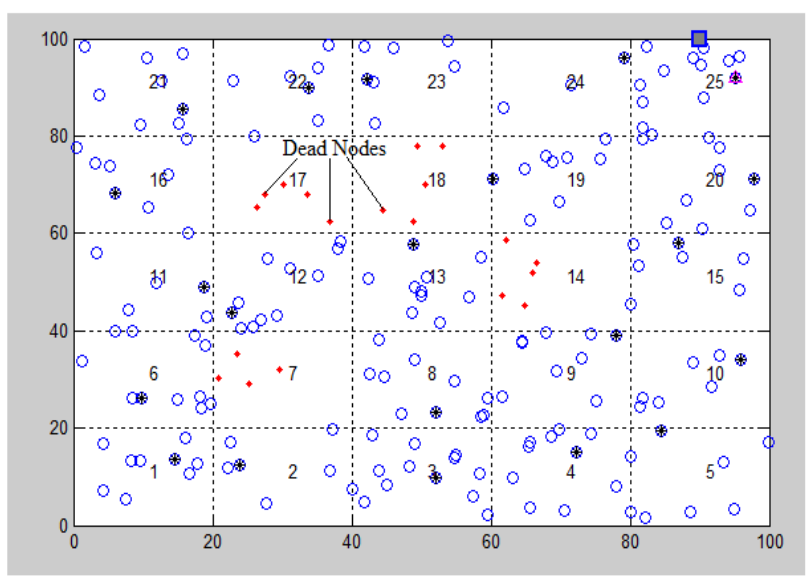

Figure 7: Network when nodes are Dead

\subsection{Fuzzy Rules}

The following figure represents the fuzzy rules as made in matlab : 
1. If (RE is LOW) and (BSdistance is CLOSE) and (NEIGHBORS is LOW) then (CHCHANCE is MLL) (1) 2. If (RE is LOW) and (BSdistance is CLOSE) and (NEGGHBORS is MEDUUM) then (CHCHANCE is VHM) (1) 3. If (RE is LOW) and (BSdistance is CLOSE) and (NEIGHBORS is HIGH) then (CHCHANCE is VLH) (1) 4. If (RE is LOW) and (BSdistance is MEDDUM) and (NEGGHBORS is LOW) then (CHCHANCE is LM) (1) 5. If (RE is LOW) and (BSDdistance is MEDUUM) and (NEGGHBORS is MEDUUM) then (CHCHANCE is M) (1) 6. If (RE is LOW) and (BSdistance is MEDUUM) and (NEIGHBORS is HIGH) then (CHCHANCE is MLLH) (1) 7. If (RE is LOW) and (BSdistance is FAR) and (NEGGHBORS is LOW) then (CHCHANCE is WL) (1) 8. If (RE is LOW) and (BSdistance is FAR) and (NEGGHBORS is MEDUUM) then (CHCHANCE is VL) (1) 9. If (RE is LOW) and (BSdistance is FAR) and (NEGGHBORS is HIGH) then (CHCHANCE is RML) (1) 10. If (RE is MEDOUM) and (BSdistance is CLOSE) and (NEEGHBORS is LOW) then (CHCHANCE is VLM) (1) 11. If (RE is MEEDUM) and (BSdistance is CLOSE) and (NEIGHBORS is MEDUUM) then (CHCHANCE is MLLM) (1) 12. If (RE is MEEDUM) and (BSdistance is CLOSE) and (NEEGHBORS is HIGH) then (CHCHANCE is RLH) (1) 13. If (RE is MEDUUM) and (BSdistance is MEDUUM) and (NEGGHBORS is LOW) then (CHCHANCE is RLLI) (1) 14. If (RE is MEDUUM) and (BSdistance is MEEDUM) and (NEIGHBORS is MEDUUM) then (CHCHANCE is RM) (1) 15. If (RE is MEDUUM) and (BSdistance is MEDUUM) and (NEGGHBORS is HIGH) then (CHCHANCE is RHM) (1) 16. If (RE is MEDUUM) and (BSdistance is FAR) and (NEGGHBORS is LOW) then (CHCHANCE is RL) (1) 17. If (RE is MEDUUM) and (BSDistance is FAR) and (NELGHBORS is MEDDUM) then (CHCHANCE is HL) (1) 18. If (RE is MEEDUM) and (BSdistance is FAR) and (NEGGHBORS is HIGH) then (CHCHANCE is VHL) (1) 19. If (RE is HIGH) and (BSdistance is CLOSE) and (NEIGHBORS is LOW) then (CHCHANCE is LH) (1) 20. If (RE is HIGH) and (BSdistance is CLOSE) and (NEIGHBORS is MEDUUM) then (CHCHANCE is HM) (1) 21. If (RE is HIGH) and (BSdistance is CLOSE) and (NEIGHBORS is HIGH) then (CHCHANCE is WH) (1) 22. If (RE is HIGH) and (BSdistance is MEDUUM) and (NEIGHBORS is LOW) then (CHCHANCE is RH) (1) 23. If (RE is HIGH) and (BSdistance is MEDUUM) and (NEIGHBORS is MEDUUM) then (CHCHANCE is H) (1) 24. If (RE is HIGH) and (BSdistance is MEDUUM) and (NEIGHBORS is HIGH) then (CHCHANCE is VH) (1) 25. If (RE is HIGH) and (BSdistance is FAR) and (NEGGHBORS is LOW) then (CHCHANCE is L) (1) 26. If (RE is HIGH) and (BSdistance is FAR) and (NEEGHBORS is MEDUUM) then (CHCHANCE is RHL) (1) 27. If (RE is HIGH) and (BSdistance is FAR) and (NEIGHBORS is HIGH) then (CHCHANCE is MHH) (1)

\section{Figure 8: Rule Base}

\subsection{Membership functions}

A membership function (MF) is a curve that defines how each point in the input space is mapped to a membership value (or degree of membership) between 0 and 1 . The input space is sometimes referred to as the universe of discourse. The only condition a membership function must really satisfy is that it must vary between 0 and 1 . The residual energy, base station distance, number of neighbors and cluster head chance membership functions are as follows :

\subsubsection{Residual energy}

The following figure shows the residual energy membership function. The membership function gives the range value for residual energy i.e between $0-0.5$.

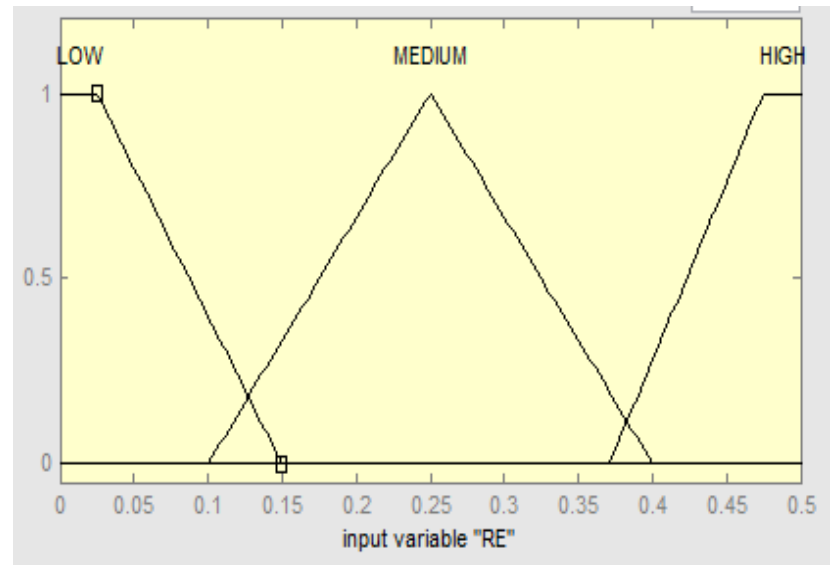

Figure 9: Residual Energy membership function

\subsubsection{Base station distance}

The following figure shows base station distance membership function. The base station may be close, medium or far from the sensor nodes.

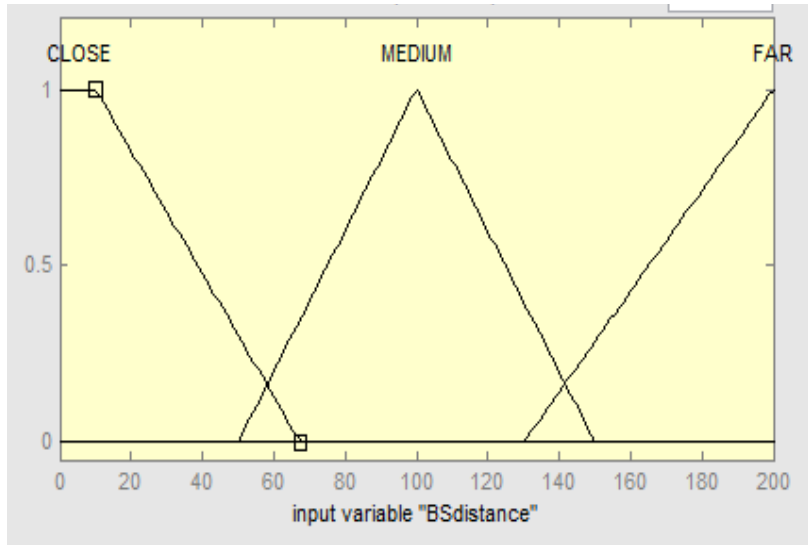

Figure 10: Base Station Distance membership function

\subsubsection{Number of neighbors}

The above figure shows membership function for the number of neighbors. The number of neighbors are taken to select the cluster head.

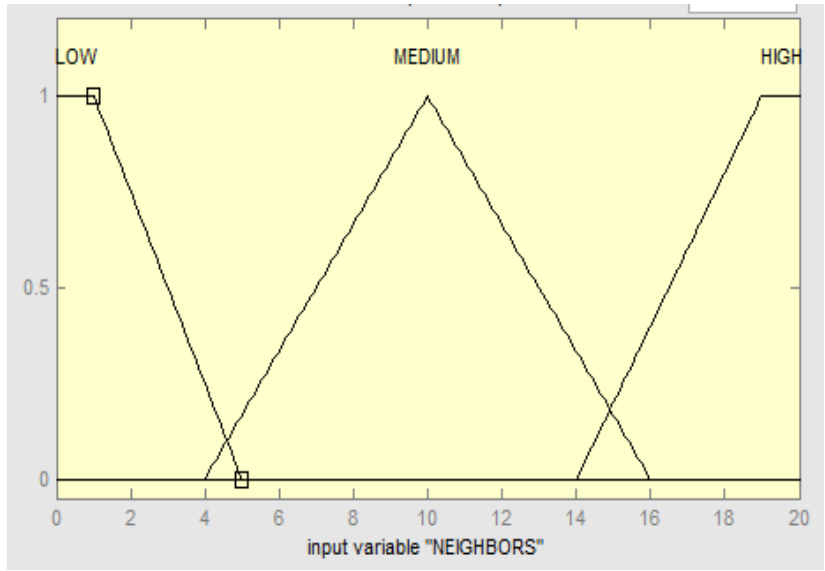

Figure 11: Number of Neighbors membership function

\subsubsection{Cluster head chance}

The following figure shows membership function for choosing the cluster head. The cluster head is chosen according to above membership functions like residual energy, base station distance and number of neighbors.

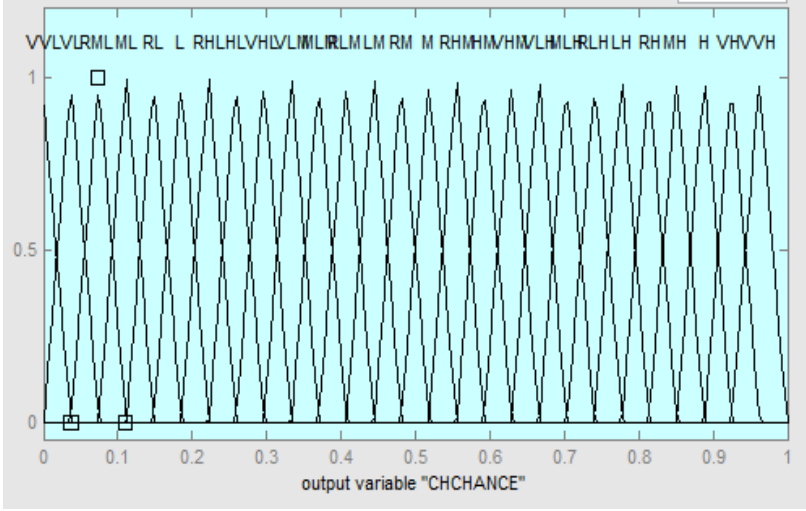

Figure 12: Cluster Head Chance membership function 


\subsection{Performance Metrics}

The performance metrics used in proposed work compared with the existing work are Network lifetime, Throughput, Average energy consumption and Network delay metrics.

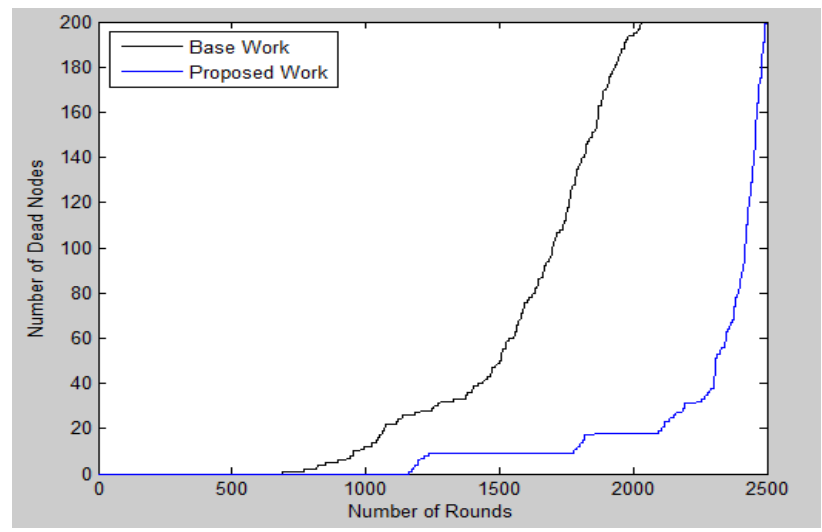

Figure 13: Analysis of dead nodes

The lifetime of network depends upon the number of Dead and Alive Nodes. Figure $13 \&$ Figure 14 clearly represents the longer stability of the proposed scheme.

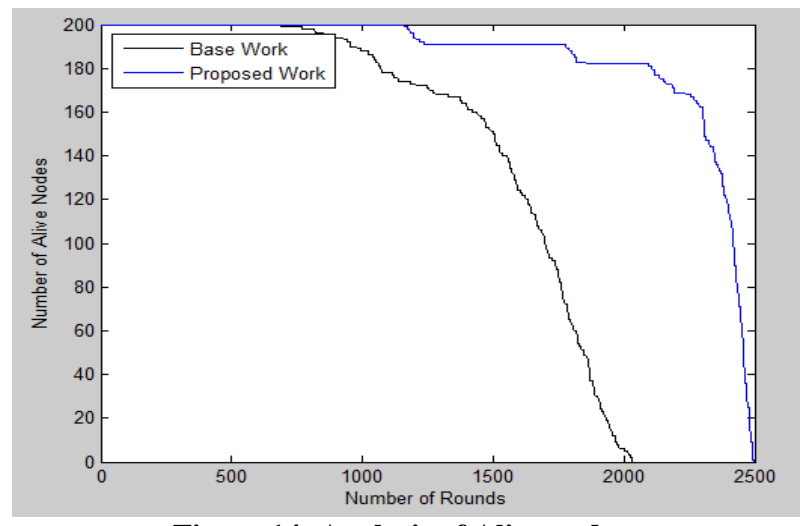

Figure 14: Analysis of Alive nodes

We can see that almost all the sensors are alive for the proposed

algorithm for quite long period of time, hence extending the network efficiency.

\subsubsection{Throughput}

It is calculated as the successful number of message delivered over a communication channel. More the Alive nodes, large number of packets are received by the destination sink node which improves the overall throughput of the network.

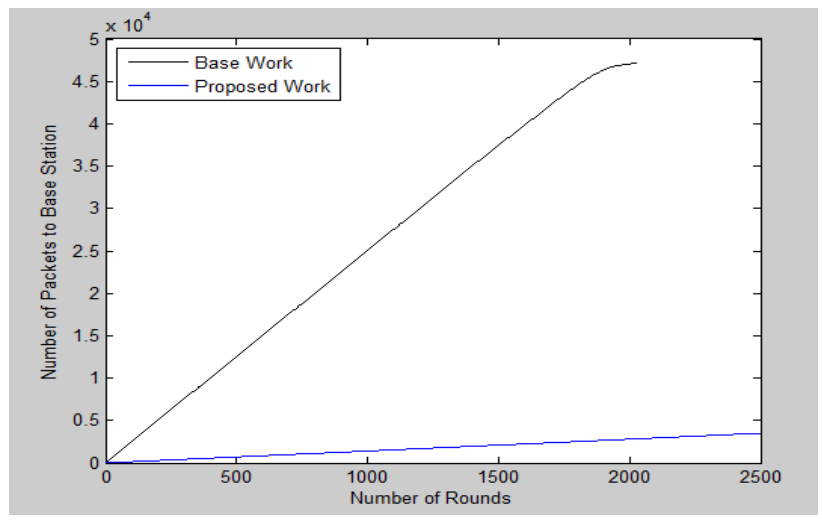

Figure 15: Throughput of the Network

\subsubsection{Energy conservation}

Figure 16 represents the average energy consumed by the network during each round of the transmission time. The average energy consumed by the proposed algorithm is minimum as compared to the existing algorithm.

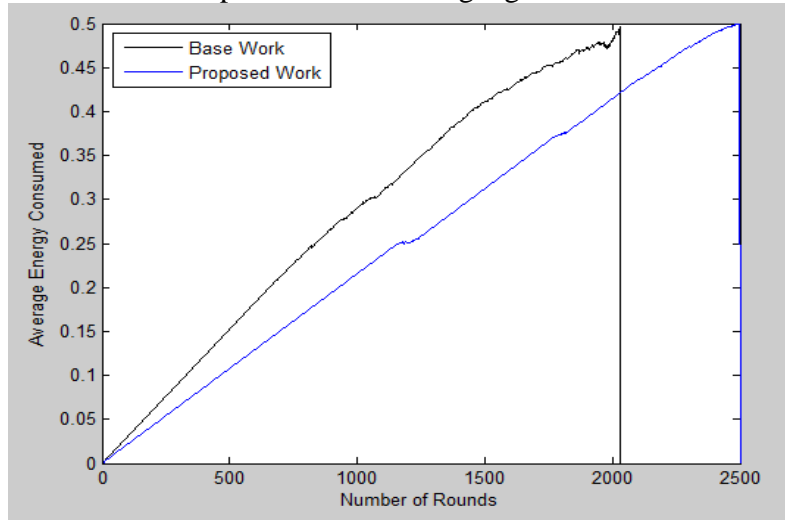

Figure 16: Energy consumed by the nodes

\subsubsection{Average remaining energy}

Figure 17 represents the average remaining energy of the network. The average remaining energy of the proposed algorithm is more as compared to the existing algorithm.

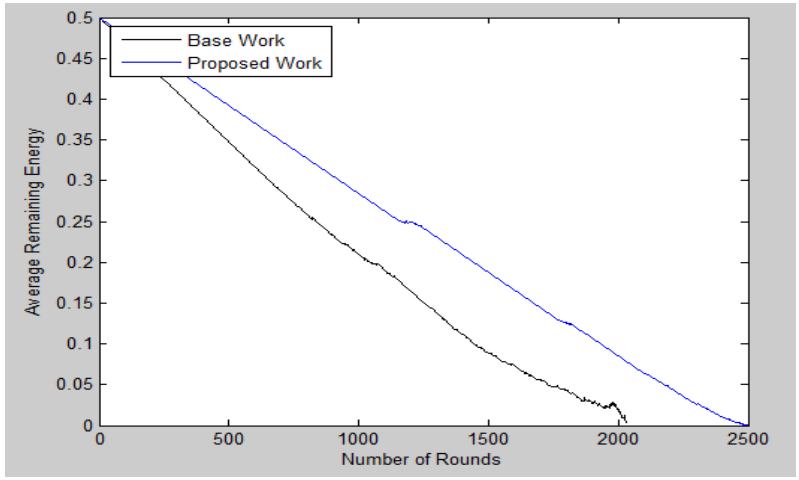

Figure 17: Average Remaining Energy

\subsubsection{Network delay}

The number of packets sent to the cluster head by the sensor nodes are available inside the figure 18 . This figure illustrates the packets sent to the cluster head in the existing work and the present work. The present work is sending more number of packets to cluster head than the existing work.

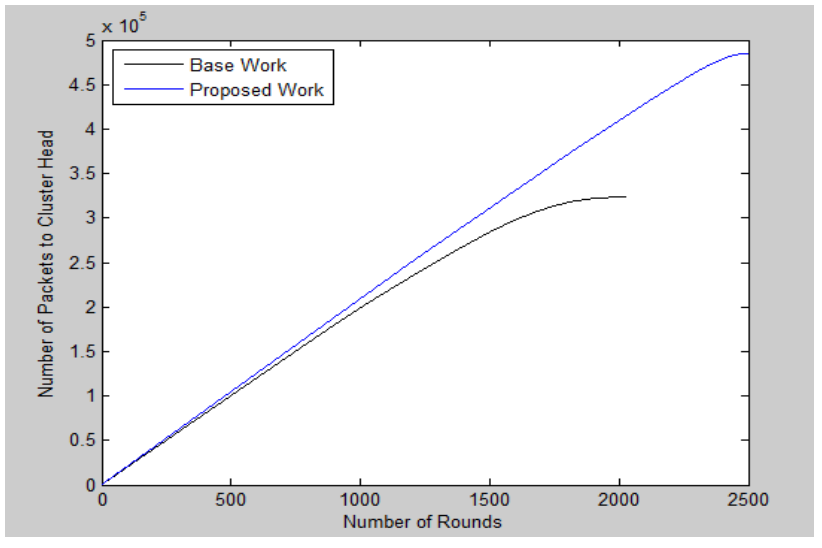

Figure 18: Network delay 


\subsection{Comparison between Existing work and proposed work}

Table 3: Table showing comparison of existing and proposed work

\begin{tabular}{|c|c|c|c|c|c|c|}
\hline $\begin{array}{c}\text { Energy } \\
=.50 J\end{array}$ & \multicolumn{3}{|c|}{ Existing work } & \multicolumn{3}{c|}{ Current work } \\
\hline S.No. & FND & MND & LND & FND & MND & LND \\
\hline 1 & 689 & 1698 & 2032 & 1162 & 2419 & 2500 \\
\hline 2 & 626 & 1693 & 2071 & 1125 & 2405 & 2496 \\
\hline 3 & 645 & 1689 & 2065 & 1150 & 2425 & 2488 \\
\hline 4 & 670 & 1655 & 1988 & 1095 & 2308 & 2348 \\
\hline 5 & 590 & 1585 & 1980 & 1202 & 2400 & 2499 \\
\hline 6 & 610 & 1650 & 2028 & 1088 & 2323 & 2382 \\
\hline 7 & 595 & 1595 & 2005 & 1130 & 2416 & 2458 \\
\hline
\end{tabular}

\section{CONCLUSIONS}

The lower cost and easier installation of the WSNs than the wired counterpart pushes industry and academia to pay more attention to this promising technology. Large scale networks of small energy-constrained sensor nodes require techniques and protocols which are scalable, robust, and energy-efficient. Hierarchy provided by clustering techniques is an efficient approach to meet such requirements. In smart cities, wireless sensor networks (WSNs) act as a type of core infrastructure that collects data from the city to implement smart services. The security of WSNs is one of the key issues of smart cities. Applications of WSNs include, controlling industrial processes, monitoring health conditions, and detecting abnormalities in the region of interest. Establishment of surveillance and security to protect the region of interest is one of the most studied applications of WSNs. This type of applications can be found in homeland security, protection of restricted facilities, battlefield surveillance, and intruder detection. Furthermore, data aggregation techniques are only performed by CHs. Our thesis work included the region based clustering, cluster head selection and energy efficient communication using mobile base station. Since it was earlier proposed that clustering improves the network lifetime. We modified the region based clustering by dividing the network area into $\mathrm{n}$ regions with cluster head chosen for each region and proposed a new method for cluster head selection having less computational complexity. It was also found that the modified approach has improved performance to that of the other clustering approaches. We have used the mobile base station with controlled trajectory path as a reference to compare the performance of each of the clustering methods. It was found that the proposed algorithm gives a much improved network lifetime as compared to existing work. In this thesis, the nodes are divided into multiple regions depending upon the network area. The region height and width can be controlled according to the requirement of the network. We virtually divide the network into regions according to the proximity to the BS denoting the closest region as the boundary zone and the farthest region as the non-boundary zone. The nodes have been classified according to the region that they fall in. Based on our model, transmission of packets for cluster-based WSNs has been proposed to balance the load among cluster heads that fall in different regions. This algorithm is applied prior to a cluster algorithm to improve the performance of the clustering algorithm without affection the performance of individual sensor nodes. As a result, the network lifetime has been prolonged. By analyzing the results and graphs, we have reached up to the solution that by changing the clustering and communication mechanism, the overall lifetime of the complete network can be improved. In all of the methods discussed above the energy parameter is taken into consideration only during cluster head selection. The performance may be increased by considering energy as a parameter during clustering itself. Multiple numbers of experiments has been conducted to evaluate the effectiveness of the experiments. Due to the potential deployment in uncontrolled and harsh environments and due to the complex architecture, wireless sensor networks are and will be prone to a variety of malfunctioning.

\section{FUTURE SCOPE}

In future work, the detection accuracy for the nodes in the Wireless Sensor Network can be calculated where detection accuracy depicts the ratio of the number of faulty sensors detected to the total number of faulty sensors in the network. In the present work, we have improved the overall lifetime of the network but the faults occur inside the WSN can be analyzed in the future in real time scenarios.

\section{ACKNOWLEDGEMENTS}

First of all I am grateful to The Almighty God for establishing me to complete this dissertation.

I'm highly indebted to a number of individuals in academic as well as in social circle who have contributed to this thesis. In particular, I wish to extend my appreciation to my supervisor Er. HARPREET KAUR, Assistant Professor at Lala Lajpat Rai Institute of Engineering and Technology, Moga, who has the attitude and substance of a genius. She continually and convincingly conveyed a spirit of adventures in regard to research and an excitement in regards to teaching. Without her guidance and persistent help this dissertation would not have been possible. Her valuable and expert supervision, attention grabbing views and obliging nature led to successful completion of this work.

I would like to take this opportunity as a special note of thanks to my parents and friends for their unceasing encouragement and support. I would not have been able to complete this thesis without their motivation.

Last but not the least; I would like to thank one and all who, directly and indirectly, have lent their helping hand in this venture and supported me in the completion of this thesis.

\section{REFERENCES}

[1] Beiranvand, Z., Patooghy, A., \& Fazeli, M. (2013, May). I-LEACH: An efficient routing algorithm to improve performance \& to reduce energy consumption in Wireless Sensor Networks. In Information and Knowledge Technology (IKT), 2013 5th Conference on (pp. 13-18). IEEE.

[2] Dastgheib, S. J., Oulia, H., \& Ghassami, M. R. S. (2011, December). An efficient approach for clustering in wireless sensor network using fuzzy logic. In Computer Science and Network Technology (ICCSNT), 2011 International Conference on (Vol. 3, pp. 1481-1486). IEEE.

[3] Gajjar, S., Sarkar, M., \& Dasgupta, K. (2014). Cluster head selection protocol using fuzzy logic for wireless sensor networks. International Journal of Computer Applications, 97(7).

[4] Kaschel, H., \& Ortega, J. (2016, October). Energy 
efficiency in routing protocols applied to WSN. In Automatica (ICA-ACCA), IEEE International Conference on (pp. 1-8). IEEE.

[5] Kumari, R., \& Nand, P. (2016, April). Performance Comparison of various Routing Protocols in WSN and WBAN. In Computing, Communication and Automation (ICCCA), 2016 International Conference on (pp. 427431). IEEE.

[6] Kushal, B. Y., \& Chitra, M. (2016, May). Cluster based routing protocol to prolong network lifetime through mobile sink in WSN. In Recent Trends in Electronics, Information \& Communication Technology (RTEICT), IEEE International Conference on (pp. 1287-1291). IEEE.

[7] Li, H., Lin, K., \& Li, K. (2011). Energy-efficient and high-accuracy secure data aggregation in wireless sensor networks. Computer Communications, 34(4), 591-597.

[8] Mohan, B. A., \& Sarojadevi, H. (2016, May). A hybrid approach for data collection using multiple mobile nodes in WSN (HADMMN). In Recent Trends in Electronics, Information \& Communication Technology (RTEICT), IEEE International Conference on (pp. 711-714). IEEE.

[9] Natarajan, H., \& Selvaraj, S. (2014). A fuzzy based predictive cluster head selection scheme for wireless sensor networks. In International Conference on Sensing Technology (pp. 560-566).

[10] Patil, M., \& Sharma, C. (2016, May). Energy efficient cluster head selection to enhance network connectivity for wireless sensor network. In Recent Trends in Electronics, Information \& Communication Technology (RTEICT), IEEE International Conference on (pp. 175179). IEEE.

[11] Pavithra, H., \& Poornima, G. R. (2016, May). An efficient mobile sink path selection approach for WSN's.
In Recent Trends in Electronics, Information \& Communication Technology (RTEICT), IEEE International Conference on (pp. 151-155). IEEE.

[12] Peng, D., \& Zhang, Q. (2010, June). An energy efficient cluster-routing protocol for wireless sensor networks. In Computer Design and Applications (ICCDA), 2010 International Conference on (Vol. 2, pp. V2-530). IEEE.

[13] Sharma, T., \& Kumar, B. (2012). F-MCHEL: fuzzy based master cluster head election leach protocol in wireless sensor network. International Journal of Computer Science and Telecommunications, 3(10), 8-13.

[14] Shukla, K. V. (2013). Research on energy efficient routing protocol LEACH for wireless sensor networks. International Journal of Engineering Research and Technology, 2(3), 1-5.

[15] Thein, M. C. M., \& Thein, T. (2010, January). An energy efficient cluster-head selection for wireless sensor networks. In Intelligent systems, modelling and simulation (ISMS), 2010 international conference on ( $\mathrm{pp}$. 287-291). IEEE.

[16] Wang, J., Yin, Y., Kim, J. U., Lee, S., \& Lai, C. F. (2012, October). A mobile-sink based energy - efficient clustering algorithm for wireless sensor networks. In Computer and Information Technology (CIT), 2012 IEEE 12th International Conference on (pp. 678-683). IEEE.

[17] Xiuwu, Y., Feisheng, F., Lixing, Z., \& Feng, Z. (2016, June). WSN monitoring area partition clustering routing algorithm for energy-balanced. In Electronics Information and Emergency Communication (ICEIEC), 2016 6th International Conference on (pp. 80-84). IEEE.

[18] Yadav, L., \& Sunitha, C. (2014). Low Energy Adaptive Clustering Hierarchy in Wireless Sensor Network (LEACH). International Journal of Computer Science and Information Technologies, 5(3), 4661-4664. 